\title{
EQUIVALENCE OF TAMELY RAMIFIED $v$-RINGS ${ }^{1}$
}

\section{NICKOLAS HEEREMA}

I. Introduction. Let $R$ be a $v$-ring, that is, an unramified complete discrete valuation ring of characteristic zero with residue field $k$ having characteristic $p \neq 0$. Let $R_{e}$ and $R_{e}^{\prime}$ be totally ramified extensions of $R$ of degree $e$. The symbol $H$ represents the natural map of a local ring on to its residue field. We say that an automorphism $\bar{\tau}$ on $k$ lifts to an automorphism $\tau$ on $R_{e}$, and $\tau$ induces $\bar{\tau}$, if $H \tau=\bar{\tau} H$. In this note we shall prove the following theorem and a number of corollaries.

Theorem 1. Assume that $(e, p)=1$ and let $\pi$ and $\pi^{\prime}$ be prime elements of $R_{e}$ and $R_{e}^{\prime}$ respectively. Then we have $p=\pi^{e} u$ and $p=\pi^{\prime} u^{\prime}$ where $u$ and $u^{\prime}$ are units in $R_{e}$ and $R_{e}^{\prime}$. If $\bar{\tau}$ is the automorphism on $k$ induced by the isomorphism $\tau: R_{e} \rightarrow R_{e}^{\prime}$ then $H\left(u^{\prime-1}\right) \bar{\tau} H(u)$ has an eth root in $k$. Conversely, if $\bar{\tau}$ is an automorphism on $k$ such that $H\left(u^{\prime-1}\right) \bar{\tau} H(u)$ has an eth root in $k$ then there exists an isomorphism $\tau$ of $R_{e}$ onto $R_{e}^{\prime}$ such that $\tau$ induces $\bar{\tau}$. Moreover, $\tau$ can be chosen so that $\tau(R)=R$.

We shall discuss a number of corollaries of Theorem 1 and defer the proof of the theorem.

CoRollary 1. An automorphism $\bar{\tau}$ on $k$ lifts to an automorphism of $R_{e}$ if and only if $H(u)^{-1} \bar{\tau} H(u)$ has an eth root in $k$.

CoROllaRY 2. If the automorphism $\bar{\tau}$ on $k$ lifts to an isomorphism $\tau$ of $R_{e}$ onto $R_{e}^{\prime}$ then $\bar{\tau}$ lifts to an isomorphism of $R_{\boldsymbol{\theta}}$ onto $R_{e}^{\prime}$ which maps $R$ onto itself.

Let $G$ denote the automorphism group of $R_{e}$ with identity mapping $\epsilon$. Let

$$
G_{t}=\left\{\alpha \mid \alpha \in G, \alpha-\epsilon\left(R_{e}\right) \subset \Pi^{t} R_{e}\right\}
$$

and

$$
H_{t}=\left\{\alpha \mid \alpha \in G_{t}, \alpha-\epsilon(\Pi) \in \Pi^{t+1} R_{e}\right\} .
$$

It is well known and not difficult to show that if $(e, p)=1$ then $H_{t}=G_{t}$ for $t>1$. Thus, in this case we have the extended chain of ramification groups

Received by the editors July 24, 1965.

1 This research was supported by NSF GP-4007. 

$\because G \supset G_{1} \supset H_{1} \supset G_{2} \supset G_{3} \supset \cdots \supset G_{i} \supset \cdots$

All the factors of (1) save $G / G_{1}$ are evaluated in [ 1 , Theorem 5]. Also, see [3, Theorem 6 and Corollary]. As an immediate consequence of Corollary 1 we have

COROLlary 3. The group $G / G_{1}$ is isomorphic to the group of all automorphisms $\bar{\tau}$ on $k$ such that $H(u)^{-1} \bar{\tau} H(u)$ has an eth root in $k$.

It was shown in the middle thirties (for a discussion, see MacLane $[3$, p. 423]) that an unramified $v$-ring is determined by its residue field. A long standing question has been the following-can one characterize the isomorphically distinct rings $R_{e}$ in terms of the structure of the residue field $k$ and if so, how? In the tamely ramified case, $(e, p)=1$, the answer is yes and the solution is given by $[1$, Theorem 3 ] in the case in which $k$ is perfect. Corollary 4 below yields the same conclusion without restriction on $k$.

As in $[1$, p. 495$]$ we consider the equivalence relation " $\sim_{e}$ " on $k^{*}$, the nonzero elements of $k$, in which $a \sim_{e} b$ if there is an automorphism $\bar{\tau}$ on $k$ such that $a^{-1} \bar{\tau}(b)$ is in $k^{e}$, the set of $e$ th powers in $k^{*}$. Let [a] represent the equivalence class containing $a$ and let $E$ be the set of all classes $[a]$.

CoRollaRy 4. The rings $R_{e}$ and $R_{e}^{\prime}$ of Theorem 1 are isomorphic if and only if $[H(u)]=\left[H\left(u^{\prime}\right)\right]$, thus the mapping $R_{e} \rightarrow[H(u)]$ induces a one to one correspondence between classes of isomorphic rings $R_{e}$ and $E$.

Proof. The first sentence follows immediately from Theorem 1. Thus the mapping $R_{e} \rightarrow[H(u)]$ is well defined, a fact which can be observed directly. Given $a \in k^{*}$ choose $u$ in $R$ such that $H(u)=a$. Thus $R_{e}=R(\pi)$, where $\pi$ is a root of $x^{e}-p u$, maps on to [a]. Thus the induced mapping is onto.

\section{Proof of Theorem 1.}

LEMMA 1. Let $R_{e}$ and $R_{e}^{\prime}$ be tamely ramified extensions of $R$ and let $\tau: R_{e} \rightarrow R_{e}^{\prime}$ be an isomorphism which induces the automorphism $\bar{\tau}$ on the residue field $k$. Then there exists an isomorphism $\eta: R_{e} \rightarrow R_{e}^{\prime}$ such that $\eta(R)=R$ and $\bar{\eta}=\bar{\tau}$.

Proof. Since every automorphism on $k$ lifts to $R$ there is an automorphism $\alpha$ on $R$ such that $\bar{\alpha}=\bar{\tau}^{-1}$. Then $\tau \alpha: R \rightarrow R_{e}^{\prime}$ has the property $\tau \alpha-\epsilon(R) \subset \pi^{\prime} R_{e}^{\prime}$. Thus, by [2, Theorem 4] $\tau \alpha$ can be extended to an automorphism $\beta$ on $R_{e}^{\prime}$ such that $\beta-\epsilon\left(R_{e}^{\prime}\right) \subset \pi^{\prime} R_{e}^{\prime}$. Now $\tau^{-1} \beta(R)$ $=\tau^{-1} \tau \alpha(R)=R$. Let $\eta=\beta^{-1} \tau$. Then we have $\eta(R)=R$ and $\bar{\eta}=\bar{\beta}^{-1} \bar{\tau}=\bar{\tau}$. 
Now, let $\tau: R_{e} \rightarrow R_{e}^{\prime}$ be an isomorphism. By Lemma 1 there exists an isomorphism $\eta: R_{e} \rightarrow R_{e}^{\prime}$ such that $\eta(R)=R$ and $\bar{\eta}=\bar{\tau}$. It follows from Theorem 3 of $\left[1\right.$, p. 494] that $H\left(u^{\prime-1}\right) \bar{\tau} H(u)$ is in $k^{e}$. The converse follows immediately from the same Theorem [1, Theorem 3] and the fact that every automorphism on $k$ lifts to $R$.

III. An example. Again we assume that $(e, p)=1$.

Using product as the operation we write $k_{e}$ for the group $k^{*} / k^{e}$. The automorphisms of $k$ induce a group $G$ of automorphisms on $k_{e}$. Let $\phi$ represent the natural map of $k^{*}$ onto $k_{e}$. For $x$ in $k_{e}$ let $[x]_{\theta}$ denote the set of elements in $k_{\theta}$ conjugate to $x$ with respect to $G$. We state without proof.

Proposition 1. Let a be in $k^{*}$. The correspondence $[a] \rightarrow[\varphi(a)]_{G}$ is $a$ one to one correspondence between $E$ and the classes of conjugate elements in $k_{e}$ with respect to $G$.

We consider the case in which $k=G F\left(p^{r}\right)$, the field with $p^{r}$ elements. Let $n=\left(e, p^{r}-1\right)$. Then for any $b$ in $k^{*}, a \sim_{e} b$ if and only if $a \sim_{n} b$. Also $k_{e}$ is the cyclic group of order $n$. Since all elements in a given conjugate class have the same order it follows that the number of conjugate classes is

$$
\sum_{q \mid n} \frac{\varphi(q)}{I(q)}
$$

where $\varphi$ is the Euler $\varphi$ function and $I(q)$ is the least positive integer $s$ such that $q \mid p^{s}-1$. We also require that $\varphi(1)=I(1)=1$. Thus, if $N(e, k)$ is the number of isomorphically distinct rings $R_{e}$ with residue field $k$, we have,

$$
N\left(e, G F\left(p^{r}\right)\right)=\sum_{o \mid\left(e, p^{r}-1\right)} \frac{\varphi(q)}{I(q)} .
$$

In particular, if $\left(e, p^{r}-1\right)=1, N\left(e, G F\left(p^{r}\right)\right)=1$, and if $\left(e, p^{r}-1\right) \mid p-1$

$$
N\left(e, G F\left(p^{r}\right)\right)=\sum_{q \mid\left(e, p^{r}-1\right)} \varphi(q) .
$$

Finally we note that the automorphisms on $k$ which lift to $R_{e}$ in the tamely ramified case are exactly those automorphisms $\alpha$ such that $\phi H(u)$ is left fixed by the mapping $\alpha$ induces on $k_{e}$. Thus every automorphism on $G F\left(p^{r}\right)$ lifts to $R_{e}$ if and only if $\left(e, p^{r}-1\right) \mid p-1$. 


\section{REFERENCES}

1. N. Heerema, On ramified complete discrete valuation rings, Proc. Amer. Math. Soc . 10 (1959), 490-496.

2. —- Inertial isomorphisms of v-rings, Canad. J. Math. (to appear).

3. S. MacLane, Subfields and automorphism groups of p-adic fields, Ann. of Math. 40 (1939), 423-442.

4. J. Neggers, Derivations on $\bar{p}$-adic fields, Trans. Amer. Math. Soc. 115 (1965), 496-504.

Florida State University 